\title{
Predictive value of clinical indices in detecting aspiration in patients with neurological disorders
}

Fabiola Mari, Monica Matei, Maria Gabriella Ceravolo, Anna Pisani, Alfeo Montesi, Leandro Provinciali

\begin{abstract}
Objectives-(1) To evaluate the predictive value of a detailed clinical screening of aspiration in patients withneurological diseases, both with and without symptoms of dysphagia taking videofluoroscopy as the gold standard; (2) to assess the existence of risk factors for silent aspiration, measuring the cost-benefit ratio of radiological examination.

Methods-93 consecutive patients meeting the diagnostic criteria for a neurological disease with a risk of swallowing dysfunctions (cerebrovascular accidents, brain injury, Parkinson's disease, multiple sclerosis, amyotrophic lateral sclerosis, myotonic dystrophy, and abiotrophic diseases) underwent a detailed clinical assessment using a 25 item form to check for symptoms of dysphagia and impairment of the oropharyngeal swallowing mechanism. The $3 \mathrm{oz}$ water swallow test was also performed to assess the aspiration risk. Sensitivity, specificity, positive predictive, and negative predictive values (NPV) of dysphagia, history of cough on swallowing, and $3 \mathrm{oz}$ test positivity, versus videofluoroscopy documented aspiration, taken as the gold standard, were measured in all the patients and in subgroups with different neurological disorders.
\end{abstract}

Results-Non-specific complaints of dysphagia showed a very poor predictive value, whereas the symptom "cough on swallowing" proved to be the most reliable in predicting the risk of aspiration, with $74 \%$ sensitivity and specificity, $71 \%$ positive predictive, and $77 \%$ negative predictive value. The standardised 3-oz test had a higher predictive potential than the clinical signs, but had low sensitivity. The association of cough on swallowing with the $3 \mathrm{oz}$ test gave a positive predictive of $84 \%$, and an negative predictive value of $78 \%$. In cases where the clinical tests failed to detect any impairment, videofluoroscopy documented only a low risk $(20 \%)$ for mild aspiration.

Conclusions-The association of two clinical items (such as history of cough on swallowing and $3 \mathrm{oz}$ test positivity) provides a useful screening tool, the cost:benefit ratio of which seems very competitive in comparison with videofluoroscopy in aspiration risk evaluation.

(F Neurol Neurosurg Psychiatry 1997;63:456-460)
Keywords: dysphagia; aspiration risk; bedside clinical assessment

Food aspiration is a frequent consequence of dysphagia, causing a strong risk of pneumonia ${ }^{1}$ and diet alterations. ${ }^{2}$ In some neurological diseases, dysphagia can persist for a long period, without being complained of by patients. $^{3}$ Therefore, a screening of swallowing alterations in such conditions is suggested, if lung infections are to be prevented.

A prospective, longitudinal cohort study, carried out on patients during the subacute phase after stroke, documented that even silent aspiration is associated with a 5.57-fold increase in risk of pneumonia. ${ }^{4}$ Videofluoroscopy showed the occurrence of a swallowing impairment in many different neurological disorders, such as cerebrovascular accidents, ${ }^{5}$ brain injury, ${ }^{6}$ Parkinson's disease, ${ }^{78}$ multiple sclerosis, ${ }^{9}$ amyotrophic lateral sclerosis, ${ }^{10}{ }^{11}$ myotonic dystrophy, ${ }^{12}$ and dementia. ${ }^{13}$

Although videofluoroscopy is, so far, the most accurate instrumental investigation to check the passage of food below the vocal folds, ${ }^{14}$ it is an invasive test that requires a specialised diagnostic laboratory with trained staff and a specific set of expensive instruments not available in all centres. ${ }^{15}$

Several clinical approaches have been proposed to make the diagnostic process easier and to measure the severity of dysphagia. ${ }^{16-18}$ In particular, clinical scales were associated with either non-specific ${ }^{19}$ or specific pathology tests $^{20}$ to predict aspiration.

The aim of this study was to evaluate the predictive value of a detailed clinical screening of aspiration in patients with neurological diseases, both with and without symptoms of dysphagia taking videofluoroscopy as the gold standard. ${ }^{21}$

Furthermore the cost:benefit ratio of the radiological examination was evaluated on the basis of such results and risk factors for silent aspiration were investigated.

\section{Methods}

PATIENTS

Ninety three consecutive patients (61 inpatients and 32 outpatients; 59 men, 34 women; mean age 59.8 (SD 16), range 18-80) admitted to the rehabilitation clinic over an 18 month period, were screened to evaluate the presence of aspiration. Inclusion criteria were as follows:

(1) presence of a neurological disorder with a documented risk of swallowing dysfunctions ${ }^{5-13}$; 
(2) informed consent to the investigation; (3) ability to cooperate. Unconscious and demented subjects, as well as bedridden patients, were excluded.

The case mix was represented by 28 acute or subacute stroke (cerebrovascular accident) (five total anterior circulation infarct, 10 partial anterior circulation infarct, four posterior circulation infarct, nine lacunar infarct, as classified according to Bamford et $a l^{2}$ ), seven recent traumatic brain injury, seven multiple sclerosis (expanded disability status scale ranging between 2.0 and 6.0, median: 3.5), 27 Parkinson's disease (Hoehn and Yahr stage ranging from 2.0 to 4.0, median: 3.0 ), six amyotrophic lateral sclerosis, five myotonic dystrophy, 13 abiotrophic diseases (four Alzheimer's disease, two multisystemic atrophy, two progressive supranuclear palsy, two olivopontocerebellar atrophy, three Friedreich diseases).

Patients with cerebrovascular accident and traumatic brain injury were examined at least one month after the clinical state had stabilised

Twenty patients $(21 \%)$ were free from symptoms of swallowing impairment. Among the others, $17(18 \%)$ complained of dysphagia for liquids, $30(32 \%)$ of dysphagia for solids, and 26 of both.

\section{PROCEDURE}

The investigation protocol was approved by the local ethics committee.

Patients underwent a detailed clinical examination based on collection of symptoms and evaluation of the oropharyngeal swallowing mechanism.

A 25 item form, drawn up on the basis of both personal experience and previous reports, ${ }^{16}{ }^{23-25}$ checked for:

(1) past or present dysphagia for either liquids or solids; (2) "knot in throat" sensation; (3) odynophagia; (4) nasal regurgitation; (5) daily episodes of cough on swallowing during the past week; (6) dysphonia; (7) dysarthria; (8) need for either feeding orthosis or nasogastric tube; (9) history of coma or pneumonia;(10) tracheostomy. The checklist also included 14 indices investigating the function of lips, tongue, mandible, and pharynx both at rest and during swallowing.

In addition, the $3 \mathrm{oz}$ water swallow test ${ }^{2026}$ was carried out by giving each patient $9 \mathrm{ml}$ water and asking them to drink from a cup without interruption. Coughing during or for up to one minute after completion or the presence of a postswallow wet or hoarse voice quality were considered positive signs of the presence of an abnormal situation.

Videofluoroscopy was used for the evaluation and identification of swallowing disorders. This examination was performed within 72 hours of the clinical examination by an observer blind to the clinical findings. ${ }^{13}{ }^{27} \mathrm{~A}$ professional video recorder providing $50 \mathrm{i} / \mathrm{s}$ frame by frame analyses, slow motion pictures (from 1/30 to five times less normal speed), and forward and backward mode, was used. Patients sitting upright were given 5 or 10 or 15 $\mathrm{ml}$ of fluid and solid barium boluses.
Table 1 Variables considered by videofluoroscopy

\begin{tabular}{ll}
\hline Swallowing phase & Radiological issues \\
\hline Oral containment & Anterior leakage \\
Posterior leakage & \\
Horizontal progression & $\begin{array}{l}\text { Deficit of horizontal transport } \\
\text { Prolonged transit time } \\
\text { Deficit of oral clearance }\end{array}$ \\
& Deficit of closure of nasal cavity \\
Pharyngeal containment & Delay of swallowing reflex \\
& Deficit of laryngeal closure \\
& Deficit of epiglottis movements \\
& Vallecular stasis \\
Vertical progression & Deficit of pharyngeal peristalsis \\
& Deficit of lingual piston \\
& Deficit of UES opening \\
& Penetration \\
& Aspiration severity \\
& Mild $<10 \%$ \\
& Moderate $<30 \%$ \\
& Severe $\geqslant 30 \%$ \\
&
\end{tabular}

The radiological examination was to assess the four phases of bolus deglutition: (1) oral containment; (2) horizontal progression (the transport from the oral cavity to the pharynx); (3) pharyngeal containment (containment, morphological and volumetrical adaptation of the pharynx, closure of the nasal, oral, and laryngeal cavities); (4) vertical progression (transport of bolus from the pharynx to the esophagus) (table 1). Aspiration was accurately assessed checking for the entry of bolus below the vocal folds. With respect to the volume of the aspirated bolus $(<10 \%, 10 \%-30 \%$, or $>30 \%$ of the total) aspiration was classified as mild, moderate, or severe respectively.

\section{DATA ANALYSIS}

Sensitivity, specificity, positive predictive, and negative predictive values of: (1) the patient's complaint of dysphagia(2); a history of cough on swallowing, and (3) positivity of the $3 \mathrm{oz}$ test versus radiologically documented aspiration taken as the gold standard, were measured in all the patients and in subgroups with different neurological disorders. The positive predictive value associated with each clinical item included in the 25 item checklist was also assessed.

Factor analysis was employed to measure the covariance of indices used to build the checklist. To compute the costs of both clinical and radiological approaches the time spent on performing the investigations and the materials employed were considered.

\section{Results}

Table 2 shows the prevalence of symptoms and signs checked for during the clinical examination and of $3 \mathrm{oz}$ test positive findings.

Videofluoroscopy documented the occurrence of aspiration in $43(46 \%)$ patients; it was mild in 25, moderate in seven, and severe in 11 . Among the remaining patients, 45 showed an impairment in at least one swallowing mechanism.

Table 2 shows the positive predictive values associated with each clinical item with respect to radiologically documented aspiration. 
Table 2 Prevalence and positive predictive value (PPV) of positive findings on the 25 item clinical assessment and of 3 oz test, used to check for aspiration risk in neurological patients

\begin{tabular}{lll}
\hline Index & $\begin{array}{c}\text { Prevalence } \\
(\%)\end{array}$ & $\begin{array}{c}P P V \\
(\%)\end{array}$ \\
\hline History collection: & & \\
Dysphagia & 78 & 52 \\
Dysarthria & 44 & 58 \\
Dysphonia & 57 & 60 \\
Knot/lump in throat & 32 & 47 \\
Nasal regurgitation & 26 & 75 \\
Cough during swallowing & 48 & 71 \\
Odynophagia & 6 & 33 \\
Coma & 10 & 67 \\
Nasogastric tube & 12 & 54 \\
Tracheostomy & 4 & 75 \\
Pneumonia & 18 & 82 \\
Clinical evaluation: & & \\
Oral phase & 30 & 68 \\
Corner deviation & 29 & 71 \\
Anterior leakage & 42 & 54 \\
Protrusion deficit & 28 & 66 \\
Drooling & 42 & 64 \\
Tongue motility impairment & 35 & 55 \\
Bolus formation deficit & 41 & 63 \\
Oral clearance impairment & 19 & 61 \\
Mandible movement deficit & 31 & 66 \\
Masseter muscle strength deficit & 17 & 57 \\
Oral sensitivity deficit & & \\
Pharyngeal phase & 60 & 54 \\
Swallow reflex impairment & 38 & 63 \\
Soft palate motility deficit & 40 & 60 \\
Hyoid-laryngeal motility & 57 \\
Pharyngeal reflex impairment & 26 & 76 \\
3 oz test positivity & 34 & \\
\hline
\end{tabular}

Symptoms of dysphagia showed a very poor predictive value. Silent aspiration occurred in four of $20(20 \%)$ patients who did not complain of any swallowing difficulty and in $49 \%$ of dysphagic patients. Among the indices in which the positive predictive value was higher than $70 \%$, history of pneumonia, tracheostomy, evidence of anterior leakage, and nasal regurgitation showed a low occurrence rate (less than 30\%).

The symptom cough on swallowing proved to be the most reliable in predicting the risk of aspiration, showing high sensitivity, specificity, positive predictive value, and negative predictive value (table 3 ). The standardised 3 oz test had a higher predictive potential than the clinical signs, but had a low sensitivity. In particular, subjects with silent aspiration gave negative findings in the $3 \mathrm{oz}$ test. For the whole population, the association of cough on swallowing with the $3 \mathrm{oz}$ test gave a positive predictive value of $84 \%$, and a negative predictive value of $78 \%$.

The analysis of subgroups showed how the correlation between symptoms of dysphagia, 3 $\mathrm{oz}$ test positivity, and aspiration differed according to the underlying neurological disease (figure), the positive predictive value of the $3 \mathrm{oz}$ test ranging from $50 \%$ in patients with

Table 3 Reliability of history collection and 3 oz test in checking for aspiration risk in neurological patients (oropharyngeal dysphagia was defined as a subjective difficulty in swallowing liquids or solids, currently or in the past)

\begin{tabular}{lllll}
\hline & $\begin{array}{l}\text { Oropharyngeal } \\
\text { dysphagia }\end{array}$ & Cough & 3 oz test & $\begin{array}{l}\text { 3 oz test } \\
+ \text { cough }\end{array}$ \\
\hline Sensitivity (\%) & 88 & 75 & 52 & 50 \\
Specificity (\%) & 30 & 74 & 86 & 91 \\
PPV (\%) & 52 & 71 & 76 & 84 \\
NPV (\%) & 75 & 77 & 68 & 76
\end{tabular}

$\mathrm{PPV}=$ positive predictive value; $\mathrm{NPV}=$ negative predictive value. traumatic brain injury to $78 \%$ in patients with a cerebrovascular accident. Silent aspiration occurred more often in patients with Parkinson's disease than in other cases.

The analysis of patients with negativity of clinical criteria produced the following "identikit":

(1) complaint of dysphagia in $30 \%$ of patients; (2) higher prevalence of Parkinson's disease $(48 \%)$ than other neurological diseases; (3) low occurrence of pneumonia, tracheostomy, coma, or feeding through nasogastric tube in the medical history (about 2\%); (4) impairment in one or more mechanisms of either oral or pharyngeal swallowing phases in $8 \%$ to $25 \%$ of patients, without any definite pattern; (5) low frequency of positive findings on videofluoroscopy; aspiration occurred in $21 \%$ patients, mostly (18\%) of a mild type and always associated with evidence of alteration in gag reflex, penetration, and vallecular stasis.

Factor analysis applied to the 25 item check list allowed the detection of seven domains, each depending on one or more clinical indices: (1) dysarthria, dysphonia, and knot or lump in the throat (2); history of coma, nasotracheal tube application, tracheostomy (3); odynophagia (4); lip corner deviation (5); dyskinesiae; (6) impairment of mandible movement (7); the association of complaints of dysphagia, nasal regurgitation, and cough on swallowing, history of pneumonia, and evidence of impairment in the oral and pharyngeal phases as detected by the checklist (anterior leakage, protrusion deficit, drooling, tongue motility deficit, bolus formation and clearance deficit, masseter strength deficit, oral sensitivity deficit, swallow and pharyngeal reflex impairment, and soft palate and hyoid-larynx motility deficit).

For the cost:benefit ratio evaluation, the bedside clinical assessment did not employ any expensive equipment and took 20 minutes on average to complete compared with the 55 minutes required by videofluoroscopy including a global radiation exposure time of up to 5.8 minutes (average 3.6 (SD 0.87) min). Both investigations were well tolerated by patients.

\section{Discussion}

The role of clinical tests in evaluating swallowing dysfunction and checking aspiration has often been discussed. Logeman ${ }^{28}$ took the amount of oral intake and the time of feeding as relevant clinical indicators of dysphagia. DePippo et $a l^{19}$ formulated the Burke dysphagia screening test, associating the $3 \mathrm{oz}$ test with other clinical signs, and administered it to 139 inpatients with stroke, concluding that the test is worthwhile in identifying patients at risk of pneumonia or airway obstruction and death. By contrast, Garon et $a l^{26}$ found the opposite in 100 patients affected by different diseases and argued that the $3 \mathrm{oz}$ test, which uses the cough reflex as the sole indicator of aspiration, is not a replacement for the precision and accuracy of videofluoroscopic evaluation.

In agreement with Fleming $e t a l^{16}$ and Garon et $a l,{ }^{26}$ the checklist employed in this study included both history and clinical indices, 


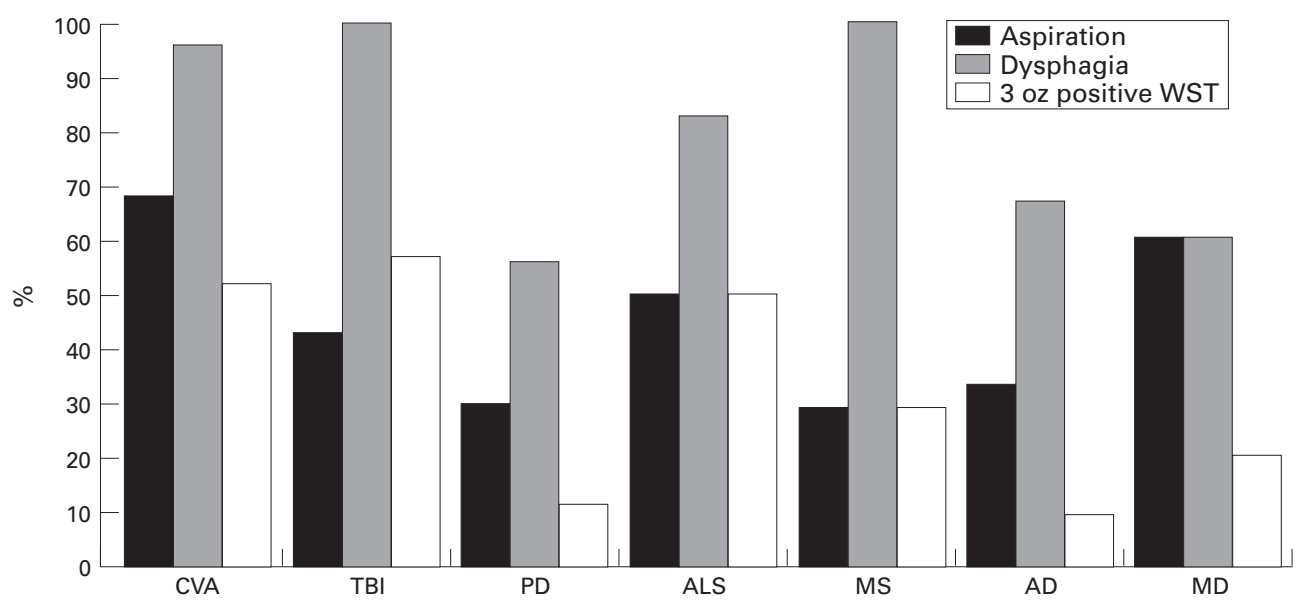

Prevalence of aspiration documented by videofluoroscopy, dysphagia, and 3 oz water swallow test (WST) test positivity in different neurological groups.

selected on the basis of knowledge of the anatomical structures and functional mechanisms involved in swallowing. Factor analysis allowed us to assess how some indices seem poorly related to impairment in swallowing mechanisms. In particular, items such as dysphonia, dysarthria, oral dyskinesiae, mandible movement deficit, and lip corner deviation, may indicate the presence of brainstem lesions, often responsible for, but not necessarily associated with swallowing impairment. ${ }^{29}$ Both sensation of a knot or lump in the throat and odynophagia may occur in neurotic syndromes and be detected in neurological patients with depressive reactions, hence being dissociated from the detection of an aspiration risk. History of coma, tracheostomy, and use of a nasogastric tube were highly correlated with each other, leading to the identification of a subgroup of patients who had severe brain lesions. Although such a condition is associated with a high risk of aspiration, ${ }^{6}$ the inclusion of the aforementioned indices in a screening assessment is not advisable, owing to their poor sensitivity.

The choice of videofluoroscopic examination as a gold standard for the detection of aspiration was made in accordance with previous investigations ${ }^{19-21} 26$; the pattern of swallowing mechanisms was assessed using a procedure already described by other authors. ${ }^{27} 30$

Patients included in this research were selected on the basis of the risk of aspiration associated with the neurological disease. The occurrence of aspiration in our patients is similar to that described in the literature for patients with cerebrovascular accident, ${ }^{29}$ traumatic brain injury, ${ }^{6}{ }^{3132}$ and multiple sclerosis. ${ }^{933}$ No definite data are available on the incidence of swallowing alterations in Parkinson's disease (rates ranging between $0 \%$ to $100 \%$ are reported), ${ }^{7} 10$ amyotrophic lateral sclerosis, myotonic dystrophy, and abiotrophic diseases. The few cases examined in this study does not allow us to draw any conclusions about the disease specific incidence rates or the higher prevalence of silent aspiration in Parkinson's disease than in other neurological groups.
The evaluation of the predictive value of the clinical examination showed that the complaint of dysphagia by the patients should be considered a non-specific feature, whatever the underlying neurological disease. Despite a prevalence of symptoms ranging from $56 \%$ to $100 \%$, true aspiration has been documented in less than one half of dysphagic patients. Although apparently low, such a percentage is still higher than those reported by Johnson et $a l^{35}$ and Perlman et $a l^{36}$ who found a prevalence of aspiration of $30 \%$ and $39 \%$ respectively, in different categories of dysphagic patients undergoing videofluoroscopy. The dissociation between severity of symptoms and swallowing alterations may partially reflect the influence of either neurological deficits (for example, poststroke facial paresis may cause oral stage dysphagia without affecting the pharyngeal stage of swallowing) or psychic problems (anxious patients may complain of swallowing alterations in the absence of any detectable deficit)

Among all the clinical signs considered, only a history of cough on swallowing proved to be really accurate in predicting aspiration, both in the total population and in subgroups with different diseases. Other conditions such as recurrent pneumonia, or previous tracheostomy, although being highly correlated with the risk of aspiration, have no clinical usefulness, owing to their low occurrence rate, as they only affect patients in severe conditions.

The 3 oz test was confirmed as a valuable tool in the evaluation of dysphagic patients, with a positive predictive value of $76 \%$ versus risk of aspiration. These findings almost overlap those described by DePippo et $a l^{20}$ in patients with stroke, by Garon et al in multiple neurological pathology groups, ${ }^{26}$ and by Splaingard et al in patients with stroke or brain injury. ${ }^{21}$

The association of history collection with objective assessment (cough plus $3 \mathrm{oz}$ test) gives an increase in both positive and negative predictive values. A similar conclusion was provided by DePippo et $a l^{19}$ who analysed the predictive significance of items included in the 
Burke dysphagia screening test and found that, in patients with stroke, "coughing associated with feeding or during a $3 \mathrm{oz}$ water swallow test could identify $92 \%$ of patients developing pneumonia or recurrent upper airway obstruction.

However, the diagnostic accuracy of the clinical evaluation depends on the preservation of the cough reflex and pharynx sensitivity; all pathological conditions which cause either an impairment in pharynx innervation or silent aspiration are unlikely to be detected by the bedside assessment and need radiological screening.

In our experience, the failure of the clinical evaluation prevented prediction of the aspiration risk in a very low percentage of patients, in whom, moreover, aspiration was always mild $(<10 \%$ bolus) and never associated with a history of lung infections. According to a recent prospective study, only aspiration of more than $10 \%$ on barium test swallows during videofluoroscopy is associated with an increased risk of pneumonia. ${ }^{4}$

In conclusion, the association of the $3 \mathrm{oz}$ test with the clinical index cough on swallowing provides a useful screening tool, the cost:benefit ratio of which seems very competitive with videofluoroscopy in evaluation of risk of aspiration. $^{37}$

Although videofluoroscopy keeps its diagnostic importance in evaluating disability after different failures of the swallowing mechanism, to plan an appropriate treatment, ${ }^{38}{ }^{39}$ the use of a detailed bedside assessment, based on a multiple item checklist, is advisable for tailoring the rehabilitation approach to each patient's needs $^{16}$ in patients in whom radiological examination is not available.

1 Horner J. Aspiration following stroke: clinical correlates and outcome. Neurology 1988;38:1359-62.

2 Martin AW. Dietary management of swallowing disorders. Dysphagia 1993;6:129-35.

3 Johnston BT, Li Q, Castell JA, Castell DO. Swallowing and esophageal function in Parkinson's disease. Am f Gastroenterol 1995;90:1741-6.

4 Holas MA, DePippo KL, Reding MJ. Aspiration and relative risk of medical complication following stroke. Arch Neurol 1994;51:1051-3.

5 Barer DH. The natural history and functional consequences of dysphagia after hemispheric stroke. $\mathcal{F}$ Neurol Neurosug of dysphagia after hemisph
Psychiatry 1989;52:236-41.

6 Yorkston KM, Honsinger MJ, Mitsuda PM, Hammen V. The relationship between speech and swallowing disorders in head-injured patients. Fournal of Head Trauma Rehabilitation 1989;4:1-16.

7 Bushmann M, Domeyer SM, Leeker L, Perlmutter JS. Swallowing abnormalities and their response to treatment in Parkinson's disease. Neurology 1989;39:1309-14.

8 Bird MR, Woodward MC, Gibson EM, Philand DJ, Fonda D. Asymptomatic swallowing disorders in elderly patient with Parkinson's disease: a description of findings on clinical examination and videofluoroscopy in sixteen patients. Age Aging 1994;23:251-4.

9 Hughes JC, Enderby PM, Langton Hewer R. Dysphagia and multiple sclerosis: a study and discussion of its nature and impact. Clin Rehabil 1994;8:18-26.

10 Robbins J. Swallowing in ALS and motor neuron disease. Neurol Clin 1987;5:213-29.
11 Welnets K. Maintaining adequate nutrition and hydratation in the dysphagia ALS patients. Canadian Nurse 1983;79: $30-4$.

12 Willing TN, Paulus J, Lecan Saint Guily J, Beon C. Swallowing problems in neuromuscolar disorders. Arch Phys Med Rehabil 1994;75:1175-85.

13 Feinberg MJ, Ekberg O, Segall L, Tully J. Deglutition in elderly patients with dementia: findings of videofluorographic evaluation and impact on staging and management. Radiology 1992;183:811-4.

14 Palmer JB, Kuhlemeier KV, Tippett DC, Lynch C. A protocol for the videofluorographic swallowing study. Dysphagia 993;8:209-14.

15 Montesi A, Pesaresi A, Antico E, Piloni V. Dynamic radiological study by videorecording of normal oropharyngeal deglutition. Radiol Med 1988;75:166-72.

16 Fleming SM, Weaver AW. Index of dysphagia: a tool for identifying deglutition problems. Dysphagia 1987;1:206-8.

17 Sonies BC, Weiffenbach J, Atkinson JC. Clinical examination of motor and sensory functions of the adult oral cavity. Dysphagia $1987 ; 1: 178-86$.

18 Martens L, Cameron T, Simonsen M. Effects of a multidisciplinar management program on neurologically impaired patients with dysphagia. Dysphagia 1990;5:147-51.

19 DePippo KL, Holas MA, Reding MJ. The Burke dysphagia screening test: validation of its use in patients with stroke. Arch Phys Med Rehabil 1994;75:1284-6.

20 DePippo K, Holas M, Reding M. Validation of 3-Oz water swallow test for aspiration following stroke. Arch Neurol 1992;42:1259-61.

21 Splaingard ML, Hutchins B, Sultan L, Chaudhuri G. Aspiration in rehabilitation patients: videofluoroscopy $v$ bedside clinical assessment. Arch Phys Med Rehabil 1988;69:63740.

22 Bamford J, Sandercock P, Dennis M, Warlow C. Classification and natural history of clinically identifiable subtypes of cerebral infarction. Lancet 1991;337:1521-6.

23 Cherney LR. Dysphagia in adults with neurologic disorders: an overview. In: Cherney LR, ed. Clinical management of dysphagia in adults and children. Gaithersburg: Aspen, 1994: $1-28$

24 Bass NH. Clinical signs, symptoms and treatment of dysphagia in the neurologically disabled. Fournal of Neurologic Rehabilitation 1990;4:227-35.

25 Layne KA, Losinski DS, Zenner PM, Ament JA. Using the Fleming index of dysphagia to establish prevalence. Dysphagia 1989;4:39-42.

26 Garon BR, Engle M, Ormiston C. Reliability of the 3-oz water swallow test utilizing cough reflex as sole indicator of aspiration. Fournal of Neurologic Rehabilitation 1995;9:13943.

27 Ekberg O. The role of radiology in the evaluation and treatment of neurologically impaired patients with dysphagia. Fournal of Neurologic Rehabilitation 1990;4:65-73.

28 Logeman JA. Evaluation and treatment of swallowing disorders. San Diego: College Hill Press, 1983:6.

29 Alberts MJ, Horner J, Gray L, Brazer SR. Aspiration after stroke: lesion analysis by brain MRI. Dysphagia 1992:7: 170-3.

30 Linden P, Kuhlmeir KV, Patterson C. The probability of correctly prediciting subglottic penetration from clinical observations. Dysphagia 1993;8:170-79.

31 Logeman J. Evaluation and treatment planning for the head injuried patient with oral intake disorders. Fournal of Head Trauma Rehabilitation 1989;4:24-33.

32 Avery Smith W, Dellarosa DM. Approaches to treating dysphagia in patients with brain injury. Am f Occup Ther 1994; 48:235-9

33 Herrera W, Zeligman BE, Gruber J, Jones MC, Pautler R, Wriston $\mathrm{R}$, et al. Dysphagia in multiple sclerosis: clinical and videofluoroscopic correlation. Fournal of Neurologic Rehabilitation 1990;4:1-8.

34 Leopold NA, Kagel MC. Prepharyngeal dysphagia in Parkinson's disease. Dysphagia 1996;11:14-22.

35 Johnson ER, McKenzie SW, Rosenquist CJ, Lieberman JS, Sievers AE. Dysphagia following stroke: quantitative evalu-
ation of pharyngeal transit times. Arch Phys Med Rehabil 1992;73:419-23.

36 Perlman AL, Booth BM, Grayhack JP. Videofluoroscopic predictors of aspiration in patients with oropharyngeal dysphagia. Dysphagia 1994;9:90-5.

37 Garon BR, Engle M, Orniston C. Silent aspiration: results of 1000 videofluoroscopic swallow evaluation. Fournal of $\mathrm{Neu}$ rologic Rehabilitation 1996;10:121-6.

38 Linden P. Videofluoroscopy in the rehabilitation of swallowing dysfunction. Dysphagia 1989;3:189-91.

39 Logemann JA. Non-invasive approaches to deglutitive aspiration. Dysphagia 1993;8:331-3. 